\title{
CARACTERIZAÇÃO DOS PROCESSOS ASSIMILATÓRIOS NO PORTUGUÊS BRASILEIRO
}

\author{
CARACTERIZACIÓN DE LOS PROCESOS DE ASIMILACIÓN EN PORTUGUÉS BRASILEÑO \\ CHARACTERIZATION OF ASSIMILATION PROCESSES IN BRAZILIAN PORTUGUESE
}

José Magalhães*

Universidade Federal de Uberlândia

\begin{abstract}
RESUMO: O objetivo principal deste artigo é descrever e mapear os fenômenos assimilatórios presentes no português brasileiro, trazendo à luz dados que justifiquem esse mapeamento, de modo a atestar que processos assimilatórios nesta língua ocorrem tanto da direita para a esquerda (assimilação regressiva) quanto da esquerda para a direita (assimilação progressiva). Procuraremos mostrar, ainda, que a assimilação progressiva envolvendo vozeamento/desvozeamento exige, muitas vezes, que o alvo seja uma consoante [+sonora]. Finalmente, trataremos também dos casos de assimilação envolvendo vogais, os quais parecem ocorrer bidirecionalmente.

PALAVRAS-CHAVE: assimilação progressiva; assimilação regressiva; português brasileiro.
\end{abstract}

RESUMEN: El propósito principal de este artículo es describir y caracterizar los fenómenos de asimilación presentes en el portugués brasileño, dando a luz datos que apoyan este mapeo con el fin de certificar que los procesos de asimilación ocurren tanto de derecha a izquierda (asimilación regresiva) cuanto de izquierda a derecha (asimilación progresiva). Trataremos de mostrar también que la asimilación de sonoridad progresiva que abarca vozeamento/desvozeamento con frecuencia exige que el objetivo sea una consonante [+sonora]. Por último, también trataremos de los casos de asimilación que abarcan vocales que parecen ocurrir de manera bidireccional.

PALABRAS-CLAVE: asimilación progresiva; asimilación regresiva; portugués brasileño.

ABSTRACT: The main purpose of this paper is to describe and map the existing assimilation phenomena in Brazilian Portuguese, showing data that support this mapping in order to certify that in this language, assimilation processes occur both from right to left (regressive assimilation) and from left to right (progressive assimilation). We aim to show also that progressive assimilation involving voicing/devoicing often requires that the target is a consonant [+voiced]. Finally, we also treat cases of assimilation involving vowels, which seem to occur bidirectionally.

KEYWORDS: progressive assimilation; regressive assimilation; Brazilian Portuguese.

\section{INTRODUÇÃO}

A assimilação, além de ser um dos processos fonológicos mais comuns nas línguas do mundo, tem sido base para justificar inúmeros modelos teóricos. Por exemplo, Clements e Hume (1995) ancoram motivações independentes para os nós da Geometria de Traços em casos de assimilação total e parcial. Borowsky (2000) avalia que a assimilação pode ser explicada com base em restrições de Fidelidade Posicional, diferenciando os processos que ocorrem no nível da palavra dos demais. Fenômenos assimilatórios são caracterizados por diferentes aspectos; um deles diz respeito à distância entre gatilho e alvo, como no Lena Asturiano (Hualde 1998; apud Walker 2004) em que vogais médias e baixas acentuadas assimilam traço de altura da vogal alta final (assimilação local), o que não acontece com vogais postônicas (assimilação de longa distância):

(1) Assimilação local

$\begin{array}{lcl}\text { kordíru } & v s & \text { kordéros } \\ \text { reúndu } & v s & \text { reóndo } \\ \text { gétu } & v s & \text { gatos }\end{array}$

(2) Assimilação de longa distância 
Burwíbanu vs burwébanos

Outro aspecto importante focaliza a direção do processo (Lombardi, 1996; Borowsky, 2000), que pode se dar da direita para esquerda - assimilação regressiva (a norma) - ou da esquerda para a direita - assimilação progressiva (rara).

Dadas essas ponderações, este artigo objetiva apresentar resultados da investigação de casos de assimilação, utilizando alguns dados do Triângulo Mineiro e da Bahia, a fim de traçar uma tipologia capaz de mapeá-los quanto à direção em que acontecem.

O português brasileiro atesta a observação de Lombardi segundo a qual a assimilação regressiva é a norma, enquanto a assimilação progressiva é a exceção. Os exemplos abaixo ilustram tais fatos:

(3) Assimilação regressiva

$$
\begin{aligned}
& \mathrm{de}[\mathrm{s}] \cdot[\mathrm{t}] \mathrm{e} \rightarrow \operatorname{de}[\mathrm{z}] \cdot[\mathrm{d}] \mathrm{e} \\
& \operatorname{asa}[\mathrm{s}][\mathrm{p}] \mathrm{retas} \rightarrow \operatorname{asa}[\mathrm{z}][\mathrm{b}] \text { rancas }
\end{aligned}
$$

(4) Assimilação progressiva ${ }^{1}$ (pós apagamento da vogal átona não final)

$$
\begin{aligned}
& \text { pê }[\mathrm{s}] \mathrm{e}[\mathrm{g}] \mathrm{o} \rightarrow \mathrm{pê}[\mathrm{s}] \cdot[\mathrm{k}] \mathrm{o} \\
& \text { có[s]e[g]as } \rightarrow \text { co[s].[k]as }
\end{aligned}
$$

Dados de fala de algumas regiões da Bahia (Hora, 1990) apontam para um terceiro caso, em que o gatilho parece estar tanto do lado esquerdo quanto do lado direito do alvo, o que, em consonância com o autor, chamaremos de assimilação bidirecional:

(5) Assimilação bidirecional
a) lei[tI]
$\sim \quad$ lei[t $[\mathrm{I}]$
$\sim \quad \operatorname{le}\left[\mathrm{t} \int \mathrm{I}\right]$
b) noi $[\mathrm{tI}]$
$\sim \operatorname{noi}\left[\mathrm{t} \int \mathrm{I}\right]$
$\sim \operatorname{no}\left[\mathrm{t} \int \mathrm{I}\right]$
c) azei[t]e
$\sim \quad$ azei[t $\mathrm{t} \mathrm{I}]$
$\sim \quad$ aze[t]I]

Juntamente com a tarefa de descrever e mapear os fenômenos assimilatórios, o presente trabalho buscará trazer à tona dados que justifiquem esse mapeamento, de modo a atestar que processos assimilatórios no português brasileiro ocorrem tanto da direita para a esquerda (assimilação regressiva) quanto da esquerda para a direita (assimilação progressiva). Procuraremos mostrar, ainda, que a assimilação progressiva envolvendo vozeamento/desvozeamento exige, muitas vezes, que o alvo seja uma consoante [+sonora]. Trataremos também dos casos de assimilação envolvendo vogais, os quais parecem ocorrer bidirecionalmente.

Antes, porém, de tratarmos dos dados do português brasileiro, apresentamos a importância dos processos assimilatórios como sustentação de modelos teóricos já consagrados da literatura.

\section{PROCESSOS DE ASSIMILAÇÃO: MODELOS TEÓRICOS}

A fonologia gerativa padrão caracterizava a assimilação em termos de cópia de traços, em que um segmento copia as especificações de traços do segmento vizinho. Na fonologia autossegmental - Geometria de Traços - ao contrário, as regras de assimilação são caracterizadas como associação (ou espraiamento) de um traço ou nó F de um segmento A para o segmento vizinho B (Clements e Hume, 1995).

Há dois tipos clássicos de assimilação: (i) preenchimento de traços, quando a regra espraia somente traços que ainda não estão especificados no alvo, o que é considerado o tipo não marcado (default); e (ii) mudança

\footnotetext{
${ }^{1}$ Dados do Banco GEFONO (Grupo de Estudos em Fonologia - Triângulo Mineiro, coordenado pelo Prof. José Magalhães - Universidade Federal de Uberlândia).
} 
de traços, quando a regra se aplica a segmentos já especificados pelo traço espraiado, substituindo seu valor original.

\subsection{Geometria de Traços (Clements e Hume 1995): espraiamento de traços}

Um dos grandes trunfos de Clements e Hume (1995) para sustentar hierarquia de nós da Geometria de Traços é a assimilação. Tomemos, como exemplo, o nó de raiz e o nó de ponto de articulação.

2.1.1 Nó de raiz: justificado por casos de assimilação total, ou seja, todas as características de um segmento se espraiam para o outro.

(6) Língua grego antigo:

\begin{tabular}{|c|}
\hline $\begin{array}{l}\text { *esmi }>\text { emmi } \\
*_{\text {*awsos }}>\text { awwos } \\
*^{*}{ }^{w} \text { olsa }>\text { bolla }\end{array}$ \\
\hline
\end{tabular}

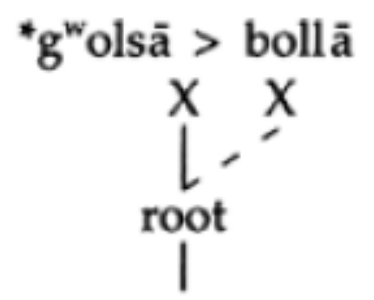

\{all features of 1$\}$

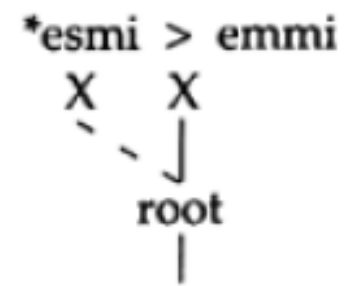

\{all features of $\mathrm{m}$ \}

(Clements e Hume, 1995:258)

2.1.2 Nó de ponto (labial, coronal, dorsal): para os autores, esses nós funcionam como uma unidade nas regras de assimilação (espraiamento).

(7) Língua Chukchi (/y/ subjacente assimila o ponto da consoante seguinte):

$\begin{array}{ll}\text { tam-pera-k } & \text { 'boa aparência' } \\ \text { ten-leut } & \text { 'boa cabeça' }\end{array}$

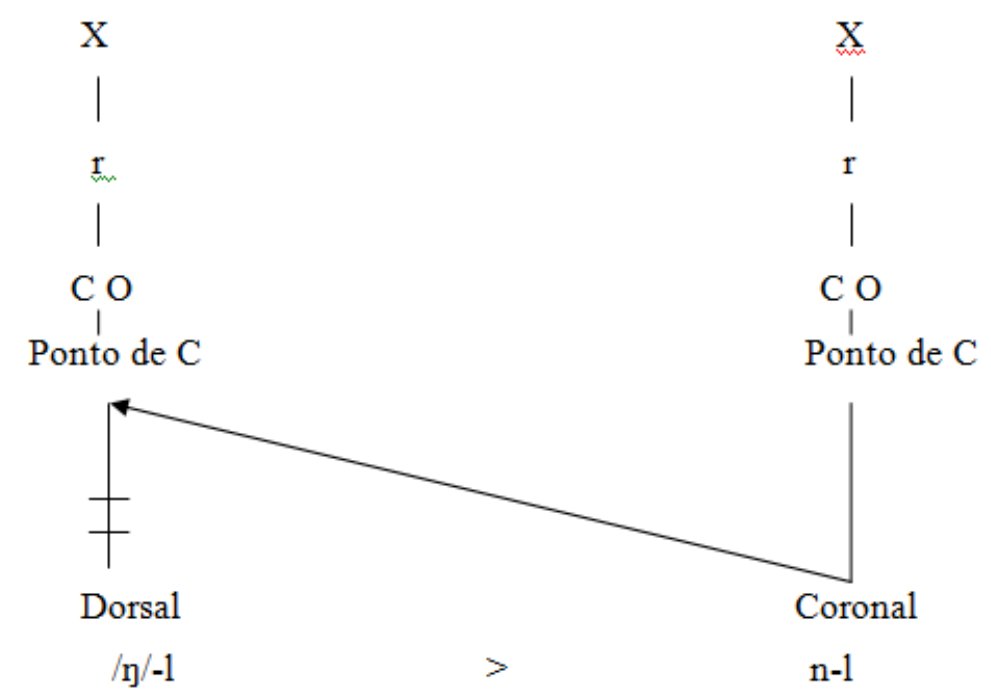




\subsection{Teoria da Otimidade - direcionalidade do processo}

2.2.1 Assimilação regressiva: fidelidade ao Onset (Lombardi, 1996)

Procurando tratar casos de assimilação regressiva e progressiva de traços de vozeamento, com base no modelo de restrições da Teoria da Otimidade, Lombardi (1996) pontua que a assimilação ocorre em satisfação à restrição AGREE a qual exige que sequências de obstruintes tenham o mesmo valor para o vozeamento.

(8) AGREE: obstruintes adjacentes concordam em vozeamento

Ressalte-se que esta restrição nada diz sobre a direção da assimilação, possibilitando, pois, que tanto a assimilação regressiva quanto a progressiva também a satisfaçam.

Segundo a autora, na avaliação do vozeamento, o traço [+sonoro] é marcado, o que é detectado pela restrição *LAR . Logo, esta restrição é violada por consoantes sonoras, mas não pelas consoantes surdas.

(9) *LAR: proibidos traços laríngeos (consoantes sonoras são proibidas)

A exigência de que haja mapeamento no output fiel ao input é formulada como uma restrição de correspondência entre input e output (IO), operando para que o vozeamento dos segmentos não mude.

(10) Ident Laryngeal (IDLAR): Consoantes devem ser fieis à especificação laríngea subjacente.

Dessa forma, a assimilação de vozeamento ocorrerá em obediência à seguinte hierarquia:

AGREE $>>$ IDLAR $>>$ * LAR, conforme ilustrado em (11).

(11) Assimilação de vozeamento

\begin{tabular}{|l|l|l|l|}
\hline pik+ben & AGREE & IDLAR & *LAR \\
\hline pikpen & & $*$ & \\
\hline pigben & & $*$ & $!^{*}$ \\
\hline pikben & $* !$ & & $*$ \\
\hline
\end{tabular}

Este tipo de assimilação se dará sempre sobre o valor não marcado para o vozeamento, independentemente da direção. Por isso, o resultado sempre será uma sequência de consoantes surdas: $\mathrm{kb} \rightarrow \mathrm{kp}$; gp $\rightarrow \mathrm{kp}$.

Para explicar que a assimilação de vozeamento é quase sempre regressiva, Lombardi (1999) introduz uma restrição de Fidelidade Posicional que focaliza o estatuto privilegiado do Onset quanto ao contraste de vozeamento. Esta restrição é Ident Onset Laryngeal (IDONSLAR) que requer fidelidade aos traços laríngeos do Onset.

(12) Ident Onset Laryngeal (IDONSLAR): o Onset deve manter-se fiel à sua especificação laríngea subjacente.

IDONSLAR domina as outras restrições de identidade de vozeamento em consoantes, mas é dominada por AGREE. Isso garante que a assimilação seja sempre regressiva, portanto $\mathrm{kb} \rightarrow \mathrm{gb}$; $\mathrm{gp} \rightarrow \mathrm{kp}$. Como se vê no tableau em (13) a seguir, o candidato ótimo é aquele que obedece aos valores laríngeos do Onset. A assimilação neste caso favorece o contraste de vozeamento marcado da consoante no Onset. 
(13)

\begin{tabular}{|c|l|l|l|l|}
\hline pik+ben & AGREE & $\begin{array}{l}\text { IDONSLA } \\
\mathrm{R}\end{array}$ & IDLAR & *LAR \\
\hline pikpen & & $* !$ & $*$ & \\
\hline Fpigben & & & $*$ & $* *$ \\
\hline pikben & $* !$ & & & $*$ \\
\hline
\end{tabular}

A restrição de Fidelidade Posicional (IDONSLAR), em seu ranqueamento normal, sempre induzirá a assimilação regressiva.

De acordo com Lombardi, a assimilação progressiva será possível somente "quando alguma outra restrição dominar os efeitos da restrição de fidelidade posicional”. Borowsky (2000) confirma este fato e aponta que o fator relevante para a assimilação progressiva é a fidelidade à palavra, o que a autora refere como WORDfaithfulness, o que apresentamos a seguir.

\subsubsection{Assimilação progressiva: fidelidade à palavra (Borowsky 2000)}

Borowsky (2000) argumenta que a assimilação progressiva de vozeamento no inglês e no holandês ocorre sistematicamente no nível da palavra, enquanto a assimilação regressiva ocorre tanto no nível da raiz quando no nível da palavra. Lembremos que, conforme aponta Lombardi (1996), a assimilação regressiva é a norma e a assimilação progressiva é rara. Ainda segundo Lombardi, a assimilação de vozeamento é geralmente regressiva. Vejamos, a seguir, a análise de Borowsky.

(14) Holandês: assimilação regressiva nos compostos (palavra+palavra):

$\begin{array}{lll}\text { /kas + buk/ } & {[\mathrm{kAzbuk}]} & \text { 'livro caixa' } \\ \text { /ka:z+pers/ } & {[\mathrm{ka}: \mathrm{spErs}]} & \text { 'prensa de queijo' } \\ \text { /goud korts/ } & {[\mathrm{xAUtkçrts]}} & \text { 'febre do ouro' } \\ \text { /le:z bril/ } & {[\text { le:zbrIl }]} & \text { 'óculos de leitura' }\end{array}$

No holandês, a assimilação progressiva ocorre nas formas do passado regular com o sufixo /-de/, em que o onset torna-se o alvo e não o gatilho da assimilação (palavra+sufixo):

(15) Holandês: assimilação progressiva em verbos no passado com sufixo -de

$\begin{array}{lll}\text { brei en } & \text { breide } & \text { 'tricotar' } \\ \text { rijmen } & \text { rijmde } & \text { 'rimar' } \\ \text { leven } & \text { levde } & \text { 'viver' } \\ \text { krabben } & \text { krabde } & \text { 'riscar' } \\ \text { stappen } & \text { stapte } & \text { 'passar' (dar passo) } \\ \text { schrappen } & \text { schrapte } & \text { 'raspar' } \\ \text { blaffen } & \text { blafte } & \text { 'descascar' }\end{array}$

Borowsky resolve este paradoxo com a restrição IDWD, a qual opera sempre em favor da identidade dos traços laríngeos na palavra.

(16) IDWD: não altere os traços da palavra

(17) Assimilação progressiva 


\begin{tabular}{|l|l|l|l|l|l|}
\hline stap+de & AGREE & IDWD & $\begin{array}{l}\text { IDONSLA } \\
\text { R }\end{array}$ & IDLAR & *LAR \\
\hline stapde & $* !$ & & & & $*$ \\
\hline stapte & & & $*$ & $*$ & \\
\hline stabde & & $* !$ & & $*$ & $* *$ \\
\hline
\end{tabular}

Como os efeitos de IDONSLAR são anulados por IDWD, AGREE é satisfeita pela assimilação progressiva do traço [-sonoro].

No caso dos compostos, cada palavra deve satisfazer IDWD. No entanto, para obedecer AGREE (dominante), uma das consoantes deve mudar, o que violará IDWD, independentemente da direção da assimilação, empatando neste ponto. A definição volta, então, ao "padrão normal regressivo", responsabilidade de IDONSLAR.

(18) Assimilação regressiva

\begin{tabular}{|l|l|l|l|l|l|}
\hline kas+buk & $\begin{array}{l}\text { AGRE } \\
\mathrm{E}\end{array}$ & IDWD & $\begin{array}{l}\text { IDONSL } \\
\text { AR }\end{array}$ & IDLAR & *LAR \\
\hline kasbuk & $* !$ & & & & $*$ \\
\hline kazbuk & & $*$ & & $*$ & $* *$ \\
\hline kaspuk & & $*$ & $* !$ & $*$ & \\
\hline
\end{tabular}

Portanto, Borowsky explica a assimilação progressiva como um efeito de fidelidade à palavra, a qual bloqueia os efeitos de fidelidade ao Onset (IDONSLAG) responsável pela assimilação regressiva.

\section{PROCESSOS ASSIMILATÓRIOS NO PORTUGUÊS BRASILEIRO}

Como apresentado nas seções anteriores, os processos assimilatórios têm servido de fundamento para sustentar a proposição de modelos teóricos autossegmentais, bem como para a motivação de hierarquia de restrições em diferentes línguas. No português brasileiro, contudo, não se tem ainda uma tipologia que categorize esses processos. Portanto, é a isto que nos propomos nas seções seguintes deste artigo.

\subsection{Direcionalidade da assimilação no português brasileiro}

\subsubsection{Consoantes}

O português brasileiro atesta a observação de Lombardi (1996), segundo a qual o processo de assimilação regressiva é o mais comum e a assimilação progressiva é rara, conforme ilustram os dados abaixo. Note-se que esses processos não se limitam ao nível da palavra.

(19) Assimilação regressiva

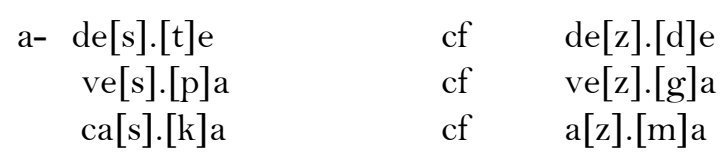




\begin{tabular}{|c|c|c|}
\hline $\begin{array}{l}\mathrm{co}[\mathrm{x}] \cdot[\mathrm{t}] \mathrm{a} \\
\mathrm{ca}[\mathrm{x}] \cdot[\mathrm{p}] \mathrm{a} \\
\mathrm{ma}[\mathrm{x}] \cdot[\mathrm{k}] \mathrm{a}\end{array}$ & $\begin{array}{l}\mathrm{cf} \\
\mathrm{cf} \\
\mathrm{cf}\end{array}$ & $\begin{array}{l}\operatorname{co}[\mathrm{\gamma}] \cdot[\mathrm{d}] \mathrm{a} \\
\mathrm{ga}[\mathrm{\gamma}] \cdot[\mathrm{b}] \text { osa } \\
\mathrm{ama}[\mathrm{\gamma}] \cdot[\mathrm{g}] \mathrm{a}\end{array}$ \\
\hline $\begin{array}{l}\text { b- } \mathrm{o}[\mathrm{s}][\mathrm{t}] \mathrm{res} \\
\text { asa[s] [p]retas }\end{array}$ & $\begin{array}{l}\mathrm{cf} \\
\mathrm{cf}\end{array}$ & $\begin{array}{l}\mathrm{o}[\mathrm{z}][\mathrm{d}] \text { ois } \\
\text { asa[z] [b]rancas }\end{array}$ \\
\hline $\begin{array}{l}\mathrm{co}[\mathrm{x}][\mathrm{p}] \text { reta } \\
\text { ma[x] [k]áspio }\end{array}$ & $\begin{array}{l}\mathrm{cf} \\
\mathrm{cf}\end{array}$ & $\begin{array}{l}\mathrm{co}[\mathrm{\gamma}][\mathrm{b}] \mathrm{ranca} \\
\mathrm{ma}[\mathrm{\gamma}][\mathrm{m}] \text { orto }\end{array}$ \\
\hline
\end{tabular}

Dados de fala informal do Banco de Dados do GEFONO (informantes do Triângulo Mineiro) comprovam que fenômenos de apagamento da vogal postônica não final em palavras proparoxítonas têm provocado o surgimento de intrigantes casos de assimilação progressiva. Esses casos envolvem a consoante da coda da penúltima sílaba resultante da síncope e o onset da sílaba final, conforme ilustrado abaixo.

(20) Assimilação progressiva

\begin{tabular}{|c|c|c|c|c|c|}
\hline \multirow[t]{3}{*}{ (a) } & pê[s]e[g]o & $\rightarrow$ & pe[s].[k]o & $\mathrm{cf}$ & *pe[z].[g]o \\
\hline & có[s]e[g] as & $\rightarrow$ & $\operatorname{co}[\mathrm{s}] .[\mathrm{k}]$ as & $\mathrm{cf}$ & ${ }^{*} \operatorname{co}[\mathrm{z}]$. $[\mathrm{g}]$ as \\
\hline & á[s]i[d]o & $\rightarrow$ & á[s].[t]o & cf & *á[z].[d]o \\
\hline \multirow[t]{3}{*}{ (b) } & fí[z]i[k]a & $\rightarrow$ & $\mathrm{fi}[\mathrm{z}] \cdot[\mathrm{g}] \mathrm{a}$ & $\mathrm{cf}$ & $\mathrm{fi}[\mathrm{s}] \cdot[\mathrm{k}] \mathrm{a}$ \\
\hline & mú[z]i[k]a & $\rightarrow$ & mú[z].[g]a & $\mathrm{cf}$ & mú[s].[k]a \\
\hline & analgé $[\mathrm{z}] \mathrm{i}[\mathrm{k}] \mathrm{o}$ & $\rightarrow$ & analgé $[\mathrm{z}] \cdot[\mathrm{g}] \mathrm{o}$ & $\mathrm{cf}$ & analgé $[\mathrm{s}][\mathrm{k}] \mathrm{o}$ \\
\hline
\end{tabular}

Confrontemos os casos de assimilação progressiva em (20a) com os dados em (21), em que não ocorre qualquer tipo de assimilação, embora o ambiente seja propício ao movimento regressivo do processo.

(21) Ausência de assimilação, mesmo com contexto propício

$\begin{array}{lllll}\text { pró[s]i[m]o } & \rightarrow & \text { pró[s].[m]o } & \text { cf } & \text { *pró[z].[m]o } \\ \text { má[s]i[m]o } & \rightarrow & \text { má[s].[m]o } & \text { cf } & \text { *má[z].[m]o } \\ \text { pé[s]i[m]o } & \rightarrow & \text { pé[s].[m]o } & \text { cf } & \text { *pé[z].[m]o } \\ \text { dé[s]i[m]o } & \rightarrow & \text { dé[s].[m]o } & \text { cf } & \text { *dé[z].[m]o } \\ \text { bú[s]o[l]a } & \rightarrow & \text { bú[s].[1]a } & \text { cf } & \text { *bú[z].[1]a } \\ \text { ví[s]e[r]a } & \rightarrow & \text { ví[s].[r]a } & \text { cf } & * \text { ví[z].[r]a } \\ \text { pá[s]a[r]o } & \rightarrow & \text { pá[s].[r]o } & \text { cf } & \text { *pá[z].[r]o }\end{array}$

Finalmente, confrontemos os casos em (20) e (21) com mais este conjunto de dados (22) em que, comprovando o que afirmamos há pouco, o ambiente descrito é perfeito para que a assimilação regressiva ocorra.

(22) Coda [+sonora] antes de onset [+soante]

$\begin{array}{lllll}\text { vigé }[\mathrm{z}] \mathrm{i}[\mathrm{m}] \mathrm{o} & \rightarrow & \text { vigé}[\mathrm{z}] \cdot[\mathrm{m}] \mathrm{o} & \text { cf. } & \text { *vigé[s].[m]o } \\ \text { centé }[\mathrm{z}] \mathrm{i}[\mathrm{m}] \mathrm{o} & \rightarrow & \text { centé }[\mathrm{z}] .[\mathrm{m}]_{\mathrm{o}} & \text { cf. } & * \text { centé }[\mathrm{s}] \cdot[\mathrm{m}] \mathrm{o} \\ \text { dí }[\mathrm{z}] \mathrm{i}[\mathrm{m}] \mathrm{o} & \rightarrow & \text { dí }[\mathrm{z}] \cdot[\mathrm{m}] \mathrm{o} & \text { cf. } & * \text { dí }[\mathrm{s}] \cdot[\mathrm{m}] \mathrm{o}\end{array}$

Nos casos em (20a), somente a assimilação progressiva é possível. Notemos, contudo que, em todos esses casos, a consoante obstruinte no onset é [+sonora]. Nos casos em (20b), com assimilação variável, todos os onsets obstruintes são [- sonoro]. Já os dados apresentados em (21) são ainda mais intrigantes, porque não admitem assimilação regressiva, embora a sequência esperada esteja presente em outras palavras, como as apresentadas em (22).

Para dar conta da análise envolvendo os casos excepcionais, ou seja, aqueles que envolvem a assimilação da esquerda para a direita, é necessário atenção especial aos casos apresentados em (20) e (21). Não é a 
pretensão deste artigo dar conta de uma classificação exaustiva da assimilação no português brasileiro, mas apenas mapear o fenômeno de forma que possa ser identificado. De todo modo, é possível afirmar que a assimilação regressiva na língua, pelo menos nos casos resultantes do apagamento da vogal postônica não final, ocorre pelo desvozeamento da consoante no onset. Essas análises, certamente, exigem um maior refinamento, mas podemos, neste momento, aventar a hipótese de que a consoante obstruinte no onset parece mais frágil do que a consoante na coda. Nos casos em que o onset é [-sonoro], detectamos em nossos dados a variação entre o processo regressivo e o progressivo (20b). Pode ser que um conjunto de dados de outras regiões mostre algo diferente, mas o que nos fornece a fala do Triângulo Mineiro respalda nossa hipótese.

Os dados apresentados em (21), embora não atestem nenhum tipo de assimilação, parecem-nos bastante instigantes porque demonstram ambientes perfeitos para a aplicação do processo de assimilação padrão. Esta afirmação encontra suporte nos exemplos apresentados em (23) a seguir, em que a fricativa na coda realiza-se como [+sonora] diante de qualquer soante.

(23) Assimilação de vozeamento da coda diante de onset [+sonoro]

(a)

a[zm]a
de[zm]aio
go[zm]a
le[zm]a
me[zm]o
quare[zm]a
O[zm]ar
espa[zm]o

3.1.2 Vogais (b)

$$
\begin{aligned}
& \text { de[zl]eal } \\
& \text { de[zl]ambido } \\
& \text { de[zl]anchar } \\
& \text { de[zl]avado } \\
& \text { i[zl]âmico } \\
& \text { I[zl]ândia } \\
& \text { Bó[zn]nia } \\
& \text { de[zl]ligar }
\end{aligned}
$$

(c)

$\operatorname{lo}[\mathrm{zn}] \mathrm{a}$, ro[zn]ar $\mathrm{a}[\mathrm{zn}] \mathrm{o}$ $\mathrm{a}[\mathrm{zn}]$ eira de[zn]utrido de[zn]atado de[zn]naturado e[zn] obar

Dados do português falado em regiões da Bahia (Hora 1990), envolvendo a vogal coronal [+alta], também nos levam a crer que casos de assimilação tanto regressiva quanto progressiva lá ocorrem. Já é bem descrito no português brasileiro que as consoantes oclusivas alveolares $/ \mathrm{t} / \mathrm{e} / \mathrm{d} /$ assimilam, em certas regiões do Brasil, os traços de altura da vogal coronal /i/, tornando-se palatalizadas, o que caracteriza um caso de assimilação regressiva, conforme ilustrado em (24).

(24) Assimilação regressiva

$\begin{array}{lll}{[\mathrm{d}] \text { inheiro }} & > & {[\mathrm{d} 3] \text { inheiro }} \\ {[\mathrm{d}] \text { itado }} & > & {[\mathrm{d} 3] \text { itado }} \\ \text { gen[t]il } & > & \text { gen[t }[\mathrm{t}] \mathrm{il} \\ \text { úl[t]imo } & > & \text { úl[t } \mathrm{t}] \text { imo }\end{array}$

Contudo, conforme atestam os dados fornecidos por Hora (1990), detectamos que este processo também ocorre na direção oposta, ou seja, da esquerda para a direita. Esta constatação pode ser bem captada a partir dos exemplos em (25) a seguir.

(25) Assimilação progressiva

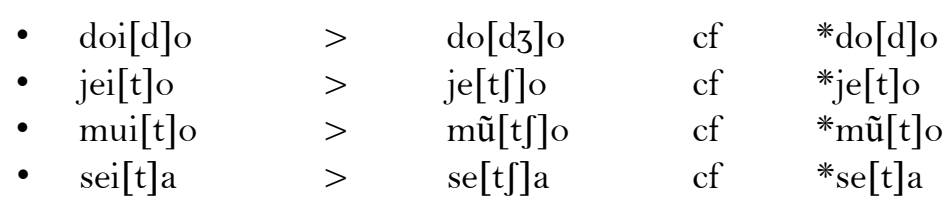


Os dados acima são reveladores de que a palatalização da consoante alveolar só pode ser explicada pela presença da vogal alta à sua esquerda, mesmo que ela não apareça na forma de superfície. Sendo este o caso, estamos diante de mais um caso de assimilação progressiva.

Um outro conjunto de dados, também fornecidos por Hora (1990), sugere a possibilidade de existir um terceiro tipo de assimilação, que o pesquisador denomina de assimilação bidirecional. A justificativa para isso estaria no fato de haver uma vogal alta à direita e outra à esquerda da consoante, não havendo motivação independente para se atestar qual estaria provocando o processo.

(26) Assimilação bidirecional
d) lei[t]e
$>$
lei[t $\left.\int \mathrm{I}\right]$
$\sim \quad \operatorname{le}\left[\mathrm{t} \int \mathrm{I}\right]$
e) noi $[\mathrm{t}] \mathrm{e}$
$>\quad$ noi[t]I]
$\sim \operatorname{no}\left[\mathrm{t} \int \mathrm{I}\right]$
f) azei[t]e
$>\quad$ azei[t]I]
$\sim \quad$ aze[t]I]

Nestes casos a bidirecionalidade se explica pelo fato de que a aplicação progressiva partiria do glide, cuja

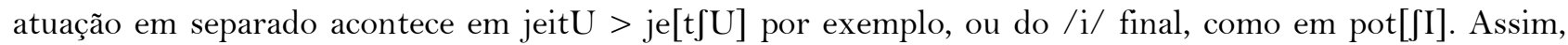
quando se tem lei[t]I] ou le[t]I] seria vago afirmar qual a direção precisa da assimilação.

\section{CONCLUSÕES}

Este artigo teve a intenção de apresentar uma proposta de mapeamento e identificação dos tipos de processos assimilatórios possíveis no português brasileiro. Sem ter como meta estabelecer uma aplicação teórica aos dados nem de tecer explicações exaustivas para o fenômeno, procuramos, inicialmente, retomar alguns modelos que se sustentam a partir da assimilação, como a Geometria de Traços (Clements e Hume 1995) e algumas hierarquias de restrições da Teoria da Otimidade (Lombardi, 1996; Borowsky, 2000 ). Referentemente aos fatos do português brasileiro, demonstramos que os casos mais comuns são mesmo os de assimilação regressiva, contudo o apagamento da vogal átona não final nas palavras proparoxítonas revelam casos explícitos de assimilação progressiva, o que ocorre com maior transparência quando o onset da sílaba final é uma obstruinte [+sonora]. Também as vogais podem estar envolvidas em fenômenos de assimilação progressiva, como acontece com a vogal coronal [+alta] no falar de algumas regiões da Bahia, provocando a palatalização da oclusiva dental a partir de traços advindos da vogal que a antecede. Para finalizar, retomamos um possível caso de assimilação bidirecional, em que a consoante que recebe os traços da vogal [+alta] posiciona-se entre duas vogais com este traço. Avaliações futuras merecem ser feitas com respeito a esses processos no português brasileiro, não apenas para realizar uma descrição mais exaustiva dos fatos, como também para se efetuarem aplicações e testagens teóricas.

\section{REFERÊNCIAS}

BOROWSKY, T. Word-faithfulness and the direction of assimilations. The Linguistic Review, Berlin, v. 17, n. $1,2000$.

CLEMENTS, G. ; HUME, E. The internal organization of speech sounds. In: GOLDSMITH, J. (Ed.). The Handbook of phonological theory. London: Blackwell, 1995.

HORA, D. da. A palatalização das oclusivas dentais: variação e representação não linear. Tese (Doutorado em Linguística Aplicada) - Faculdade de Letras, Pontifícia Universidade Católica do Rio Grande do Sul, Porto Alegre, 1990.

LOMBARDI, L. Restrictions and direction of voicing assimilation: an OT account. University of Maryland Working Papers in Linguistics, v. 3, p.89-115, 1996.

WALKER, R. Vowel feature licensing at a distance: evidence from northern Spanish language varieties. Chand, V. et al (ed.). Proceedings of WCCFL 23, 2004. p. 787-800. 